\title{
EXTREMAL SOLUTIONS OF A CLASS OF NONLINEAR INTEGRO-DIFFERENTIAL EQUATIONS IN BANACH SPACES
}

\author{
HONG-KUN XU AND JUAN J. NIETO
}

(Communicated by Palle E. T. Jorgensen)

\begin{abstract}
The monotone iterative technique is applied to a class of nonlinear first order integro-differential equations in Banach spaces. First a linear system with a "small" nonlinear perturbation is solved using Banach's Contraction Principle and the technique of Green's function. Then based upon a comparison result, the existence of minimal and maximal solutions is proved.
\end{abstract}

\section{INTRODUCTION}

We are concerned with the periodic boundary value problem (PBVP) for the following first order integro-differential equation:

$$
\left\{\begin{array}{l}
u^{\prime}(t)=f(t, u(t), K u(t)), \quad t \in I:=[0, T], \\
u(0)=u(T)
\end{array}\right.
$$

in a partially ordered Banach space $X$, where $T$ is a positive number, $f: I \times X \times$ $X \rightarrow X$ is a continuous function, and $K: C[I ; X] \rightarrow C[I ; X]$ is the bounded linear integral operator determined by a kernel $k \in C(J)$ with $J=I \times I$; that is,

$$
[K x](t)=\int_{0}^{T} k(t, s) x(s) d s, \quad x \in C[I ; X] .
$$

Integro-differential equations find many applications in various mathematical problems; see Corduneanu's book [1] and references therein for details. In this paper we shall apply the monotone iterative technique (cf. [4]) to the PBVP (1.1) to prove the existence of its extremal solutions on the interval $I$. In the next section we prove the existence of a unique solution of a linear system with a "small" nonlinear perturbation using the techniques of Green's function and Banach's Contraction Principle. After a comparison result is established in Section 3, we are able in the final section to prove the existence of minimal and maximal solutions of the PBVP

Received by the editors March 13, 1996.

1991 Mathematics Subject Classification. Primary 45J05; Secondary 34G20.

Key words and phrases. Periodic boundary value problem, first order nonlinear integrodifferential equation, Green's function, monotone iterative technique, minimal and maximal solutions, normal cone, Banach space .

Part of the paper was announced by the first author at the International Symposium on Methods and Applications of Analysis, City University of Hong Kong, Hong Kong, December 16-19, 1994.

The first author was partially supported by the South African Foundation for Research Development. 
(1.1). The reader is referred to [3] for a class of integro-differential equations of Volterra type in the one-dimensional case.

\section{A LineAR SYSTEM WITH NONLINEAR PERTURbation}

Consider the system

$$
\left\{\begin{array}{l}
u^{\prime}(t)+M u(t)=g(t, u(t),[K u](t)), \quad t \in I, \\
u(0)=u(T)+\lambda,
\end{array}\right.
$$

where $M \in \mathbb{R} \backslash\{0\}, g \in C[I \times X \times X ; X], \lambda \in X$, and $K$ is the operator defined by (1.2). Denote by $\|K\|$ the norm of $K$ and by $\|k\|_{\infty}$ the norm of the kernel $k$ (i.e., $\left.\|k\|_{\infty}=\max \{|k(t, s)|: t, s \in I\}\right)$. Then it is immediate that

$$
\|K\| \leq T\|k\|_{\infty} .
$$

Write

$$
G(t, s)=\frac{1}{1-e^{-M T}} \begin{cases}e^{-M(t-s)} & \text { if } 0 \leq s \leq t \leq T \\ e^{-M(T+t-s)} & \text { if } 0 \leq t \leq s \leq T\end{cases}
$$

Note that $G(t, s)$ is the Green function for the linear periodic boundary value problem:

$$
u^{\prime}(t)+M u(t)=\sigma(t), \quad u(0)=u(T),
$$

where $\sigma \in C[I ; X]$. Now it is not hard to see that $u \in C[I ; X]$ is a solution of $(2.1)$ if and only if

$$
u(t)=\int_{0}^{T} G(t, s) g(s, u(s),[K u](s)) d s+h_{\lambda}(t)
$$

where

$$
h_{\lambda}(t)=\frac{\lambda e^{-M t}}{1-e^{-M T}} .
$$

Let $S$ be the operator defined by the right-hand side of (2.2). It is easily seen that $S$ maps continuously $C[I ; X]$ into $C[I ; X]$, and $u \in C[I ; X]$ is a solution of $(2.1)$ if and only if it is a fixed point of $S$. Hence we can use fixed point theory to solve Eq. (2.1). We suppose that $g$ satisfies a Lipschitz condition:

$$
\|g(t, x, y)-g(t, u, v)\| \leq L_{1}\|x-u\|+L_{2}\|y-v\|, \quad t \in I, x, y, u, v \in X .
$$

Theorem 1. Assume (2.3) and

$$
\|k\|_{\infty}<\frac{|M|-L_{1}}{T L_{2}} .
$$

Then Eq. (2.1) has a unique solution in $C[I ; X]$.

Proof. We shall show that under (2.3) and (2.4), the operator $S: C[I ; X] \rightarrow C[I ; X]$ is a contraction; hence Banach's Contraction Principle yields a unique fixed point 
of $S$, which is then the unique solution of (2.1). We have for all $u, v \in C[I ; X]$,

$$
\begin{aligned}
\|[S u](t)-[S v](t)\| & \leq \int_{0}^{T}|G(t, s)| \cdot\|g(s, u(s), K u(s))-g(s, v(s), K v(s))\| d s \\
& \leq \int_{0}^{T}|G(t, s)|\left(L_{1}\|u(s)-v(s)\|+L_{2}\|[K u](s)-[K v](s)\|\right) d s \\
& \leq\left(L_{1}+L_{2}\|K\|\right) \int_{0}^{T}|G(t, s)| \cdot\|u(s)-v(s)\| d s \\
& \leq \frac{L_{1}+L_{2}\|K\|}{|M|}\|u-v\|_{\infty} \quad\left(\text { as } \int_{0}^{T}|G(t, s)| d s=\frac{1}{|M|} \text { for } t \in I\right) \\
& \leq \frac{L_{1}+T L_{2}\|k\|_{\infty}}{|M|}\|u-v\|_{\infty},
\end{aligned}
$$

which shows that $S: C[I ; X] \rightarrow C[I ; X]$ is a contraction as $\left(L_{1}+T L_{2}\|k\|_{\infty}\right) /|M|<$ 1. This completes the proof.

An immediate consequence of Theorem 1 is the following result which is due to Liz and Nieto [6] for the case $X=\mathbb{R}$, the real line.

Corollary 1. For any $\sigma \in C[I ; X], \lambda \in X, M \in \mathbb{R} \backslash\{0\}$, and $N \in \mathbb{R}$, the linear system

$$
\left\{\begin{array}{l}
u^{\prime}(t)+M u(t)=-N[K u](t)+\sigma(t), \quad t \in I, \\
u(0)=u(T)+\lambda
\end{array}\right.
$$

has a unique solution $u \in C[I ; X]$ provided $\|k\|_{\infty}<\frac{|M|}{|N| T}$.

\section{A COMPARISON RESUlT}

In order to implement the monotone iterative technique to the PBVP (1.1), we need to establish a comparison result for the linear system (2.5). First we recall some preliminaries about ordered Banach spaces. Let $X$ be a Banach space. By a cone in $X$ we mean a nonempty closed convex subset $V$ of $X$ with the properties:

(i) $\lambda V \subseteq V$ for all $\lambda \geq 0$, and

(ii) $V \cap(-V)=\{0\}$.

If $V$ is a cone in $X$, it then induces a partial ordering " $\leq$ " on $X$ by

$$
x \leq y(x, y \in X) \Longleftrightarrow y-x \in V .
$$

Recall that a cone $V$ in $X$ is said to be normal if there exists some constant $\nu>0$ such that

$$
0 \leq x \leq y \Longrightarrow\|x\| \leq \nu\|y\| .
$$

We now establish the following comparison result, which will play a crucial role when we apply in the next section the monotone iterative technique to the PBVP (1.1). To do so, we assume that $X$ is a partially ordered Banach space whose ordering " $\leq$ " is given by a cone $V$. We set

$$
C^{+}[I ; X]=\{u \in C[I ; X]: u(t) \geq 0, t \in I\} .
$$

Let $X^{*}$ be the dual space of $X$. We note that $u \in C^{+}[I ; X]$ if $x^{*} u(t) \geq 0$ for every $t \in I$ and every $x^{*} \in X^{*}$ such that $x^{*}(x) \geq 0$ for all $x \in V$. Note also that condition (2.4) implies that we require $L_{1}<|M|$. Indeed, if $L_{1} \geq|M|$, then (2.1) 
is not uniquely solvable in general; for example, take $g(t, u, v)=\sigma(t)+M u$ with $\sigma \in C[I ; X], \lambda=0$, so that (2.1) reads

$$
u^{\prime}(t)=\sigma(t), \quad t \in I ; \quad u(0)=u(T) .
$$

This problem possesses at least one solution (and hence infinitely many solutions) if and only if $\int_{0}^{T} \sigma(s) d s=0$.

Theorem 2. Assume $M, N \in \mathbb{R}$ with $M>0, \sigma \in C^{+}[I ; X]$ and $\lambda \in V$. Assume also

$$
\|k\|_{\infty} \leq \frac{M^{2}}{|N|\left(e^{M T}+M T-1\right)}=: c .
$$

Then the linear system (2.5) has a unique solution $u \in C^{+}[I ; X]$.

Proof. Since $c<M /|N| T$, Eq. (2.5) has a unique solution $u \in C[I ; X]$ by Theorem 1. So it remains to show the positivity of $u$. To this end, we first assume $X=\mathbb{R}$, the real line. Since the operator $S$ is a contraction on $C[I ; X]$, the sequence $\left\{S^{n} w\right\}$ of the Picard iterates converges strongly to the solution $u$, where

$$
w(t)=\int_{0}^{T} G(t, s) \sigma(s) d s+h_{\lambda}(t)
$$

After manipulations of the iterates $S^{n} w$ for $n \geq 1$, we find that the solution $u$ can be expressed in the form (cf. [6] for more details in the one-dimensional case):

$$
u(t)=\int_{0}^{T} H(t, s) \sigma(s) d s+\int_{0}^{T} Q(t, s) h_{\lambda}(s) d s+h_{\lambda}(t),
$$

where

$$
\begin{gathered}
H(t, s)=\int_{0}^{T} Q(t, r) G(r, s) d r+G(t, s), \\
Q(t, s)=\sum_{n=1}^{\infty} R^{(n)}(t, s),
\end{gathered}
$$

with $R^{(1)}(t, s)=R(t, s)$ and

$$
R^{(n)}(t, s)=\int_{0}^{T} R^{(n-1)}(t, r) R(r, s) d r, \quad n \geq 2,
$$

being the iterated kernels of

$$
R(t, s):=-N \int_{0}^{T} G(t, r) k(r, s) d r .
$$

We have the estimates:

$$
\begin{gathered}
\|R\|_{\infty} \leq \frac{|N|\|k\|_{\infty}}{M} \leq \frac{|N| c}{M}=: d<\frac{1}{T}, \\
\left\|R^{(n)}\right\|_{\infty} \leq(d T)^{n-1} d, \quad n=1,2, \ldots, \\
\|Q\|_{\infty} \leq \sum_{n=1}^{\infty}\left\|R^{(n)}\right\| \leq \frac{d}{1-d T} \leq \frac{|N| c}{M-|N| c T} .
\end{gathered}
$$


It follows that

$$
\begin{aligned}
H(t, s) & \geq-\|Q\|_{\infty} \int_{0}^{T} G(t, s) d s+\min _{(t, s) \in J} G(t, s) \\
& =-\frac{\|Q\|_{\infty}}{M}+\frac{1}{e^{M T}-1} \\
& \geq-\frac{|N| c}{M(M-|N| c T)}+\frac{1}{e^{M T}-1}=0, \\
\int_{0}^{T} Q(t, s) h_{\lambda}(s) d s+h_{\lambda}(t) & \geq-\|Q\|_{\infty} \int_{0}^{T} h_{\lambda}(s) d s+\min _{t \in I} h_{\lambda}(t) \\
& =-\frac{\lambda}{M}\|Q\|_{\infty}+\frac{\lambda e^{-M T}}{1-e^{-M T}} \\
& \geq \lambda\left(-\frac{|N| c}{M(M-|N| c T)}+\frac{1}{e^{M T}-1}\right)=0 .
\end{aligned}
$$

Now from expression (3.2) we conclude that $u(t) \geq 0$ for all $t \in I$. The general case where $X$ is a Banach space can be reduced to the above special case. Indeed, let $x^{*} \in X^{*}$ be such that $x^{*}(x) \geq 0$ for all $x \in V$ and let $u^{*}(t)=x^{*} u(t)$. Thus $u^{*}$ is the solution of the system

$$
\left\{\begin{array}{l}
u^{\prime}(t)+M u(t)=-N[K u](t)+\sigma^{*}(t), \quad t \in I, \\
u(0)=u(T)+\lambda^{*}
\end{array}\right.
$$

in $\mathbb{R}$, where $\sigma^{*}:=x^{*} \sigma \in C^{+}(I)$ and $\lambda^{*}:=x^{*}(\lambda) \geq 0$. From the above-proved case it follows that $x^{*} u(t) \geq 0$ for all $t \in I$. This finishes the proof.

\section{The monotone iterative technique}

In this section we shall use the monotone iterative technique to prove the existence of minimal and maximal solutions of the PBVP (1.1). Assume that $X$ is an ordered Banach space whose partial ordering " $\leq$ " is defined by a normal cone $V$. We shall say that a function $u \in C[I ; X]$ is a lower solution of the PBVP (1.1) if

$$
u^{\prime}(t) \leq f(t, u(t), K u(t)), \quad t \in I,
$$

and

$$
u(0) \leq u(T) .
$$

An upper solution $v \in C[I ; X]$ for the PBVP (1.1) is similarly defined by reversing the inequalities above. If $u, v \in C[I ; X]$ are such that $u(t) \leq v(t), t \in I$, then the interval $[u, v]$ denotes the set

$$
\{w \in C[I ; X]: u(t) \leq w(t) \leq v(t), t \in I\} .
$$

We need certain hypotheses on the lower and upper solutions of the PBVP (1.1), the function $f$, and the kernel $k$.

(H1) $u_{0}, v_{0}$ are lower and upper solutions of the PBVP (1.1), respectively, such that $u_{0}(t) \leq v_{0}(t)$ for all $t \in I$.

(H2) Let $f \in C[I \times X \times X ; X]$ satisfy a one-sided Lipschitz condition:

$$
f(t, x, y)-f(t, u, v) \geq-M(x-u)-N(y-v)
$$

whenever $t \in I, u_{0}(t) \leq u \leq x \leq v_{0}(t)$, and $\left[K u_{0}\right](t) \leq v \leq y \leq\left[K v_{0}\right](t)$, where $M, N \in \mathbb{R}$ are positive numbers. 
(H3) Whenever $A$ is a nonempty bounded set in $C[I ; X]$,

$$
\alpha(f(t, A(t), K A(t)) \leq L \alpha(A(t)), \quad t \in I,
$$

for some constant $L \geq 0$ which is independent of $A$, where $A(t)=\{x(t)$ : $x \in A\}, K A(t)=\{K x(t): x \in A\}$, and $\alpha$ is Kuratowski's measure of noncompactness in $X$ defined as follows (cf. [2] for details):

$\alpha(B)=\inf \{\varepsilon>0: B$ admits a finite cover by sets of diameter less than $\varepsilon\}$,

where $B$ is a nonempty bounded set of $X$.

(H4) $k \in C^{+}(J)$ is such that $\|k\|_{\infty} \leq c$, with $c$ being given by (3.1).

We now state and prove the main result of the paper.

Theorem 3. Assume (H1)-(H4). Then there exist two sequences $\left\{u_{n}\right\}_{n=1}^{\infty}$ and $\left\{v_{n}\right\}_{n=1}^{\infty}$ in $C[I ; X]$ satisfying

$$
u_{0} \leq u_{1} \leq \cdots \leq u_{n} \cdots \leq v_{n} \cdots \leq v_{1} \leq v_{0}
$$

such that $\left\{u_{n}\right\},\left\{v_{n}\right\}$ uniformly converge on $[0, T]$ to the minimal solution $\rho$ and the maximal solution $r$, respectively, of the PBVP (1.1) in the interval $\left[u_{0}, v_{0}\right]$.

Proof. For each $\eta \in\left[u_{0}, v_{0}\right]$, consider the linear PBVP

$$
\left\{\begin{array}{l}
u^{\prime}(t)+M u(t)=-N K u(t)+\sigma_{\eta}(t), \\
u(0)=u(T),
\end{array}\right.
$$

where

$$
\sigma_{\eta}(t)=M \eta(t)+N[K \eta](t)+f(t, \eta(t),[K \eta](t)) .
$$

Let $A \eta$ be the unique solution of Eq. (3.1) which is guaranteed by Corollary 1 as $\|k\|_{\infty} \leq c<M / N T$. Then $A$ defines a map from $\left[u_{0}, v_{0}\right]$ into $C[I ; X]$. We now show that

(i) $A u_{0} \geq u_{0}$ and $A v_{0} \leq v_{0}$, and

(ii) $\forall \eta_{1}, \eta_{2} \in\left[u_{0}, v_{0}\right], \eta_{1} \leq \eta_{2} \Longrightarrow A \eta_{1} \leq A \eta_{2}$.

Before proving (i) and (ii), we remark that (i) and (ii) imply the invariance of the interval $\left[u_{0}, v_{0}\right]$ under the map $A$.

To prove (i) we write $u=A u_{0}$ and $v=u-u_{0}$. Then $u$ satistifes Eq. (4.1) with $\eta=u_{0}$. First we observe that

$$
v(0)=u(0)-u_{0}(0)=u(T)-u_{0}(0) \geq u(T)-u_{0}(T)=v(T) ;
$$

thus $v(0)=v(T)+\lambda$ with $\lambda \in V^{+}$. From Eq. (4.1) (with $\eta=u_{0}$ ) it follows that

$$
\begin{aligned}
v^{\prime}(t)+M v(t)+N K v(t)= & u^{\prime}(t)+M u(t)+N K u(t) \\
& -\left[u_{0}^{\prime}(t)+M u_{0}(t)+N K u_{0}(t)\right] \\
= & \sigma_{u_{0}}(t)-\left[u_{0}^{\prime}(t)+M u_{0}(t)+N K u_{0}(t)\right] \\
\geq & \sigma_{u_{0}}(t)-\sigma_{u_{0}}(t)=0
\end{aligned}
$$


as $u_{0}$ is a lower solution of the PBVP (1.1). It then follows from Theorem 2 that $u \geq u_{0}$. That $A v_{0} \leq v_{0}$ is proved similarly. We now turn to (ii). To this end we set $u_{i}=A \eta_{i}(i=1,2)$ and $v=u_{2}-u_{1}$. It is easy to see that $v(0)=v(T)$. Moreover, from (4.1), (4.2) and (H2) it follows that

$$
\begin{aligned}
v^{\prime}(t)+M v(t)+N K v(t)= & \sigma_{\eta_{2}}(t)-\sigma_{\eta_{1}}(t) \\
= & M\left[\eta_{2}(t)-\eta_{1}(t)\right]+N K\left[\eta_{2}(t)-\eta_{1}(t)\right] \\
& +f\left(t, \eta_{2}(t),\left[K \eta_{2}\right](t)\right)-f\left(t, \eta_{1}(t),\left[K \eta_{1}\right](t)\right) \\
\geq & 0 .
\end{aligned}
$$

Again by Theorem 2, $v(t) \geq 0$ for all $t \in I$. This proves (ii).

Now we recursively define the sequences $\left\{u_{n}\right\}$ and $\left\{v_{n}\right\}$ in $\left[u_{0}, v_{0}\right]$ by

$$
u_{n}=A u_{n-1}, \quad v_{n}=A v_{n-1}, \quad n \geq 1
$$

By (i) and (ii) above, we see that $\left\{u_{n}\right\}$ is increasing while $\left\{v_{n}\right\}$ is decreasing, and $u_{n} \leq v_{n}$ for each $n \geq 1$. We claim that $\left\{u_{n}\right\}$ and $\left\{v_{n}\right\}$ uniformly converge on $I$. Toward this, we write

$$
B(t)=\left\{u_{n}(t)\right\}, \quad B^{\prime}(t)=\left\{u_{n}^{\prime}(t)\right\}, \quad m(t)=\alpha(B(t)), \quad t \in I
$$

First we show that $\left\{u_{n}\right\}$ is equicontinuous on $I$. By the definition of $u_{n}$, it is not hard to see that $u_{n}$ can be written as

$$
u_{n}(t)=e^{-M t}\left\{y_{n}+\int_{0}^{t} \sigma_{n}(\tau) e^{M \tau} d \tau\right\}
$$

where

$$
y_{n}=\frac{1}{e^{M T}-1} \int_{0}^{T} \sigma_{n}(\tau) e^{M \tau} d \tau
$$

$$
\sigma_{n}(t)=-N\left[K u_{n}\right](t)+M u_{n-1}(t)+N\left[K u_{n-1}\right](t)+f\left(t, u_{n-1}(t),\left[K u_{n-1}\right](t)\right) .
$$

From (4.4) it follows that the boundedness of $\left\{u_{n}\right\}$ in $C[I ; X]$ implies the equicontinuity of $\left\{u_{n}\right\}$. Since $\left\{u_{n}\right\} \subset\left[u_{0}, v_{0}\right]$ and the cone $V$ is normal, $\left\{u_{n}\right\}$ is indeed bounded in $C[I ; X]$. Let $w_{n}(t)=f\left(t, u_{n}(t), K u_{n}(t)\right)$. As $f(t, x, y)+M x+N y$ is increasing with respect to both $x$ and $y$, we have

$$
f\left(t, u_{0}(t),\left[K u_{0}\right](t)\right) \leq w(t) \leq f\left(t, v_{0}(t),\left[K v_{0}\right](t)\right), \quad t \in I
$$

which yields the boundedness of $\left\{w_{n}\right\}$ in $C[I ; X]$ again as the cone $V$ is normal. It then follows from (4.5) that $\left\{\sigma_{n}\right\}$ is bounded in $C[I ; X]$ and hence $\left\{u_{n}\right\}$ is equicontinuous. 
We now estimate the Dini derivative $D^{+} m(t)$ :

$$
\begin{aligned}
D^{+} m(t) & =\lim _{r \downarrow 0} \sup _{0 \leq h \leq r} \frac{m(t+h)-m(t)}{h} \\
& \leq \lim _{r \downarrow 0} \sup _{0 \leq h \leq r} \alpha\left(\left\{\frac{u_{n}(t+h)-u_{n}(t)}{h}\right\}\right) \\
& \leq \lim _{r \downarrow 0} \sup _{0 \leq h \leq r} \alpha\left(\bigcup_{n=1}^{\infty} \overline{c o}\left\{u_{n}^{\prime}(s): t \leq s \leq t+h\right\}\right) \\
& =\lim _{r \downarrow 0} \alpha\left(\bigcup_{n=1}^{\infty} \overline{c o}\left\{u_{n}^{\prime}(s): t \leq s \leq t+r\right\}\right) \\
& \leq \lim _{r \downarrow 0} \alpha\left(\overline{c o}\left(\bigcup_{n=1}^{\infty}\left\{u_{n}^{\prime}(s): t \leq s \leq t+r\right\}\right)\right) \\
& =\lim _{r \downarrow 0} \alpha\left(\bigcup_{n=1}^{\infty}\left\{u_{n}^{\prime}(s): t \leq s \leq t+r\right\}\right) \\
& =\lim _{r \downarrow 0} \alpha\left(\left\{B^{\prime}(s): t \leq s \leq t+r\right\}\right) .
\end{aligned}
$$

Since it is readily seen from (4.1) that

$$
\begin{aligned}
B^{\prime}(t) \subseteq & -M B(t)-N K(B(t))+M B(t) \\
& +N K(B(t))+f(t, B(t), K(B(t))),
\end{aligned}
$$

it then follows from the last inequality above that

$$
\begin{aligned}
D^{+} m(t) \leq & \lim _{r \downarrow 0}\left[2 M \alpha\left(\bigcup_{t \leq s \leq t+r} B(s)\right)+2 N \alpha\left(\bigcup_{t \leq s \leq t+r} K(B(s))\right)\right. \\
& \left.+\alpha\left(f\left(I, \bigcup_{t \leq s \leq t+r} B(s), K\left(\bigcup_{t \leq s \leq t+r} B(s)\right)\right)\right)\right] \\
\leq & \left(2 M+2 N\|k\|_{\infty}+L\right) \lim _{r \downarrow 0} \alpha\left(\bigcup_{t \leq s \leq t+r} B(s)\right) .
\end{aligned}
$$

The equicontinuity of $\left\{u_{n}\right\}$ then leads to the conclusion

$$
D^{+} m(t) \leq \ell m(t), \quad t \in I,
$$

where $\ell=2 M+2 N\|k\|_{\infty}+L$. Since 0 is the only solution to the linear periodic problem

$$
u^{\prime}(t)=\ell u(t), \quad t \in I, \quad u(0)=u(T),
$$

it follows from (4.6) that $m(t) \equiv 0$ on $I$ as $m(0)=m(T)$; see [5] for related comparison results. Hence $\left\{u_{n}\right\}$ is relatively compact in $C[I ; X]$ and admits a subsequence converging to some $\rho \in C[I ; X]$. Again because the cone $V$ is normal, the full sequence $\left\{u_{n}\right\}$ itself converges to $\rho$ in $C[I ; X]$. Via the same argument, we can show that $\left\{v_{n}\right\}$ converges to some $r \in C[I ; X]$. Now it easily follows from (4.3) that $\rho$ and $r$ are solutions of the PBVP (1.1). Finally we show that $\rho$ and $r$ are the minimal and maximal solutions of the PBVP (1.1) in the interval $\left[u_{0}, v_{0}\right]$, 
respectively. So assume that $u$ is any solution of (1.1) in $\left[u_{0}, v_{0}\right]$. By induction, we see that

$$
u_{n} \leq u \leq v_{n} \text { for all } n \geq 0 .
$$

Therefore, passing to the limit as $n \rightarrow \infty$, we arrive at the desired conclusion: $\rho \leq u \leq r$. The proof is complete.

Hypothesis (H3) is a type of compactness condition, and from the proof of Theorem 3 given above, we find that it is only used to prove the relative compactness in $C[I ; X]$ of the sequences $\left\{u_{n}\right\}$ and $\left\{v_{n}\right\}$ defined by (4.3). We will see that if the cone $V$ or the space $X$ satisfies some additional conditions, then hypothesis (H3) can be removed from Theorem 3. We recall that a cone is said to be regular if every monotone increasing sequence bounded above is (strongly) convergent. A regular cone must be normal (cf. [2]). If the cone $V$ is regular, then it has been proved (cf. [7, Lemma 2.2]) that every monotone sequence in $C[I ; X]$ equicontinuous on $I$ must converge uniformly on $I$. Hence the result below follows.

Theorem 4. If the cone $V$ is regular, then Theorem 3 holds without hypothesis (H3).

Recall now that a cone is called weakly regular provided every monotone increasing sequence bounded above is weakly convergent. Since it is not hard (cf. [7] for details) to prove that a cone that is both normal and weakly regular is regular, we arrive at the following result.

Theorem 5. If the cone $V$ is normal and weakly regular, then Theorem 3 is valid without hypothesis (H3).

A Banach space $X$ is said to be weakly sequentially complete if every weak Cauchy sequence in $X$ (i.e. a sequence $\left\{x_{n}\right\}$ of $X$ such that $\lim _{n \rightarrow \infty} x^{*}\left(x_{n}\right)$ exists for all $\left.x^{*} \in X^{*}\right)$ is weakly convergent. It is then straightforward that a reflexive space is weakly sequentially complete. By Lemma 2.3 of [7], a normal cone in a weakly sequentially complete space is weakly regular. Therefore we have the following consequence of Theorem 5 .

Corollary 2. If $X$ is weakly sequentially complete (reflexive, in particular), then the conclusion of Theorem 3 is true without hypothesis (H3).

\section{ACKNOWLEDGMENT}

The first author wishes to thank his colleague at UWD, Professor Erwin Brüning, for helpful comments on the manuscript.

\section{REFERENCES}

1. C. Corduneanu, Integral Equations and Applications, Cambridge University Press, 1991. MR 92h: 45001

2. K. Deimling, Nonlinear Functional Analysis, Springer-Verlag, Berlin/Heidelberg, 1985. MR 86j: 47001

3. S. Hu and V. Lakshmikantham, Periodic boundary value problems for integro-differential equations of Volterra type, Nonlinear Analysis: TMA 10 (1986), 1203-1208. MR 88c:45009

4. G.S. Ladde, V. Lakshmikantham and A.S. Vatsala, Monotone Iterative Techniques for Nonlinear Differential Equations, Pitman Advanced Publishing Program, 1985. MR 88g:35006

5. V. Lakshmikantham and S. Leela, Differential and integral inequalities, vols I, II, Academic Press, New York, 1969. MR 52:837; MR 52:838 
6. E. Liz and J.J. Nieto, Periodic boundary value problem for integro-differential equations with general kernel, Dynamic Systems and Applications 3 (1994), 297-304. MR 95f:45005

7. H.K. Xu, M. Su and X.W. Lu, The monotone iterative technique for first order differential equations in Banach spaces, Math. Japonica 38 (1993), 667-673. MR 94d:34072

Department of Mathematics, University of Durban-Westville, Private Bag X54001, Durban 4000, South Africa

E-mail address: hkxu@pixie.udw.ac.za

Departamento de Análisis Matemático, Facultad de Matemáticas, Universidad de Santiago de Compostela, 15706 Santiago de Compostela, Spain

E-mail address: amnieto@usc.es 